\title{
The Prevalence of Mental Health Problems and Associated Risk Factors among Military Health Workers of COVID-19 Specialized Hospitals in Wuhan, China: A Cross-Sectional Survey
}

\section{Xiao Pan}

Changzheng Hospital

\section{Ying Xiao}

Hongkong district of Changhai Hospital

\section{Ding Ren}

PLA Navy No.905 Hospital

\section{Zhengmei Xu}

Changzheng Hospital

\section{Qian Zhang}

Changhai Hospital

\section{Liying Yang}

Changhai Hospital

Fen Liu

Changhai Hospital

\section{Yushi Hao}

Changzheng Hospital

Yonghai Bai ( $\nabla$ baiyonghai179@126.com )

Shanghai Changzheng Hospital https://orcid.org/0000-0001-5818-7216

\section{Feng Zhao}

Changhai Hospital

\section{Research}

Keywords: Coronavirus Disease 2019, mental health, depressive symptoms, generalized anxiety, somatic symptoms, risk factor

Posted Date: April 30th, 2020

DOI: https://doi.org/10.21203/rs.3.rs-22537/v1 
License: (c) (i) This work is licensed under a Creative Commons Attribution 4.0 International License. Read Full License 


\section{Abstract}

Background China has been severely affected by COVID-19 (Coronavirus Disease 2019) since December 2019.In the combat against COVID-19, military health workers in China suffered from many pressures. This study aimed to investigate the current psychological status and risk factors of the military health workers.

Methods Using a web-based cross-sectional survey, we collected data from 194 military health workers from three inpatient wards in two COVID-19 specialized hospitals. The survey questions consisted of demographic information, Patient Health Questionnaire-9 (PHQ-9), Generalized Anxiety Disorder-7 (GAD7) and Patient Health Questionnaire-15 (PHQ-15). Hierarchical regression analysis was used to explore potential risk factors for mental health problem.

Results The overall prevalence of depressive symptoms, generalized anxiety and somatic symptoms were $37.6 \%, 32.5 \%$ and $50 \%$, respectively. Severe depression, generalized anxiety and somatic symptoms was $5.2 \%, 3.6 \%$ and $15.5 \%$. In $22.7 \%$ of cases, comorbidities existed between depression, generalized anxiety and somatization. Junior-grade professional title was associated with depression, older age was associated with generalized anxiety and somatization, and less sleep duration and poor sleep quality were associated with all three symptoms.

Conclusion The prevalence of depression, generalized anxiety and somatic symptoms were high in military health workers of COVID-19 specialized hospitals during the COVID-19 outbreak. Junior-grade professional title, older age, less sleep duration, and poor sleep quality have significant effects on the mental health of military health workers. Continuous surveillance and monitoring of the psychological consequences of the COVID-19outbreak should become routine to promote the mental health of military health workers.

\section{Background}

A novel pneumonia of unknown reason was reported in Wuhan, Hubei Province in December 2019. On February 11, 2020, the World Health Organization (WHO) named this disease as Coronavirus Disease 2019 (COVID-19) as it was caused by a novel coronavirus[1]. As of February 22, 2020, there are 76,936 confirmed cases and 4,148 suspected cases in the mainland China. Health workers faced mounting psychological pressures due to the massive amount of confirmed and death cases during the outbreak of COVID-19.

The first-line health workers were bearing inconceivable workload and huge risk of infection while saving lives from the threat of COVID-19. According to Wang and his colleagues' research, infected health workers accounted for 29 percent of all hospitalized COVID-19 patients in the early stage ofCOVID-19[2]. Besides, Kang and his team's research reported that they experienced psychological distress on account of social isolation and possible discrimination[3]. Reported by Panagioti and his colleagues, mental health problems caused by such psychological distress tend to impair their attention, cognitive function 
and clinical decision-making[4], increasing the possibility of medical negligence. Consequently, the mental health of health workers should be paid more attention during the COVID-19 outbreak.

Among all the health workers, those from the military are a special group deployed by the central government to fight the combat. On January 24,2020 , military health workers arrived in Wuhan as the first group from other parts of China to carry out medical rescue under the circumstance of inadequate equipment and little known about COVID-19. Since military health workers began to receive patients at COVID-19 specialized hospital on February 4, 2020, they have been suffering from more pressures including patients' concentration, the requirements of military discipline, and high-intensity work, etc. Therefore, it is necessary to focus on the mental health of this special population.

Depression, generalized anxiety and somatic symptoms are common symptoms of medical staff and patients under stress, and the comorbidity rate exceeds 50\% [5]. Several studies have explored the psychological effect during such epidemics, such as Severe Acute Respiratory Syndrome (SARS) in 2003. Sixty-eight percent of first-line health workers in a study directed by Koh reported severe pressure related to work and $57.0 \%$ experienced psychological disturbance during SARS outbreak[6]. Another study showed $17.3 \%$ significant mental symptoms among health care workers during SARS epidemic [7].A survey was conducted in 255 Chinese breast cancer patients reports the percentage of high somatic symptoms was $43.5 \%$ [8]. In consequence, depression, general generalized and somatic symptoms should be paid more attention during the outbreak of COVID-19.

This study aimed to investigate the psychological status and risk factors of first-line military health workers so as to provide targeted interventions on the mental health for military health workers during the COVID-19 and future similar epidemic outbreaks.

\section{Methods}

A questionnaire survey has been used for data collection. This cross-sectional survey was conducted during February 7, 2020 to February 21, 2020. Two hundred military health workers who had worked two to four weeks for rescuing COVID-19 patients and chosen by cluster sampling from three inpatient wards in two COVID-19 specialized hospitals were recruited. The inclusion criteria were a) military health workers, b) working in COVID-19 specialized hospitals, and c) no cognitive impairment. The exclusion criterion were a) the answer time for all 31-items questions was more than 30 minutes or less than 1 minutes, b) with schizophrenia, severe mood disorders and other mental disorders. The questionnaires were provided and collected by using "Wen Juan Xing", one professional online questionnaire platform in China.

A nine-item Patient Health Questionnaire (PHQ-9) Scale selected from the Diagnostic and Statistical Manual of Mental Disorders V (DSM-V) was used to measure depression symptoms. Each item was rated from 0 (never) to 3 (nearly every day), representing the degree to which an individual has been bothered by depression over the past two weeks. The PHQ-9 score ranged from 0-27, and higher scores indicated more depression severity. Total scores of 5, 10, 15, and 20 represented cutoff points for low, medium, 
moderate-sever, and high depression symptom severity, respectively. Based on a research done by Teymoori and his team, a PHQ-9 score $\geqq 5$ represented the existence of depression symptoms[5]. Cronbach's alpha was 0.815 .

Generalized Anxiety Disorder-7 (GAD-7) Scale selected from the Diagnostic and Statistical Manual of Mental Disorders-V (DSM-V) was used to measure the anxiety symptoms. Seven items assessed the frequency of anxiety symptoms over the past two weeks on a 4-point Liker-scale ranging from 0 (never) to 3 (nearly every day). Higher scores indicated more severe functional impairments as a result of anxiety. Total scores of 5,10 , and 15 represented cutoff points for low, medium, and high anxiety symptom severity, respectively. According to a research done by Toussaint and colleagues, A GAD-7 score $\geqq 5$ represented the existence of anxiety symptoms[6]. Cronbach's alpha was 0.896 .

Patient Health Questionnaire-15 (PHQ-15) Scale selected from the Diagnostic and Statistical Manual of Mental Disorders-V (DSM-V) was used to measure the severity of somatic symptoms. The PHQ-15 comprises 15 somatic symptoms from the PHQ, each symptom scored from 0 ("not bothered at all") to 2 ("bothered a lot"). Total scores of 5, 10, and 15 represented cutoff points for low, medium, and high somatic symptom severity, respectively. Carno-Garcia and colleagues' study indicated that a PHQ-15 score $\geqq 5$ represents the existence of somatic symptoms[7]. Cronbach's alpha was 0.862 .

Items in the questionnaire had been modified after a pilot survey by the researchers. Data were then collected through anonymous online surveys which were accomplished via WeChat. For this survey, there is a positive vote by the ethics panel of the Medical Association Changzheng Hospital (No.2020SL010).All subjects in this study has given their informed consent.

Only one response per IP address to the questionnaire was permitted during the investigation. The questionnaire could be submitted only after all items in it have been filled in. Meanwhile, background monitoring was conducted in the real time to ensure the data reliability.

Statistical analyses were performed with IBM SPSS Statistics 21.0.Descriptive analyses were conducted to describe the demographic characteristics and the overall prevalence for military health workers in depression, generalized anxiety and somatic symptoms. Also, t-test, one-way variance (ANOVA) and nonparametric analysis were used to compare different prevalence of depression, generalized anxiety and somatic symptoms between different subgroups. Hierarchical regression analysis was applied to identify independent variables associated with depression, generalized anxiety and somatic symptoms among the military health workers respectively. $P<0.05$ was considered as statistically significant.

\section{Results}

A total of 194 military health workers were recruited in the current study, among which $158(81.4 \%)$ were females. In term of age, $102(52.6 \%)$ were under 30 years old, 87 (44.8\%) were between 30 and 50, 5 (2.6\%) were over 50 . And 42 (21.6\%), 148 (76.3\%) and 4 (2.1\%) were doctors, nurses, command staff, respectively. One hundred and ten (56.7\%) have junior-grade professional title, $56(28.9 \%)$ for the medium- 
grade professional title and $28(14.4 \%)$ for the senior-grade. Frequency of daily checking news about COVID-19 > 10times were 23 (11.9\%), and 3-10 times were 93 (47.9\%). The average daily working hours $\geq 8$ hours were $61(31.4 \%)$, the average daily sleep duration $<6$ hours were $36(18.6 \%)$, and those who had ordinary or bad sleep quality were 157 (80.9\%), see Table 1.

Table 2 reports the average score and overall prevalence of depression, generalized anxiety and somatic symptoms assessed with PHQ-9, GAD-7 and PHQ-15. As shown in Table 2, the average score of PHQ-9, GAD-7 and PHQ-15 were 3.79 $\pm 3.30,3.09 \pm 3.22$ and $5.34 \pm 4.44$, respectively. As the cut-off score was 5 , the overall prevalence of depression, generalized anxiety and somatic symptoms was $37.6 \%, 32.5 \%$ and $50 \%$, respectively. As the score $\geq 10$ was defined as severe symptoms, prevalence of severe depression, severe generalized anxiety and severe somatic symptoms was $5.2 \%, 3.6 \%$ and $15.5 \%$, respectively. In $22.7 \%$ of cases, comorbidities existed between depression, generalized anxiety and somatization.

The score of depression, generalized anxiety and somatic symptoms under different subgroups was presented in the Table 3. In terms of depression, participants with junior $(p=0.003)$ and medium $(p=$ 0.012) grade professional titles had significantly higher score than those with senior titles. Those military health workers with daily average news checking of more than 10 times had significantly higher score than those who checked 3 to 10 times $(p=0.009)$. Participants who had sleep duration per day $\geq 6 \mathrm{~h}(\mathrm{p}<$ $0.001)$ and good sleep quality $(p<0.001)$ were less likely to generate depression symptoms.

In terms of anxiety, participants with junior $(p=0.049)$ and medium $(p=0.006)$ grade professional titles had significantly higher score than those with senior titles. Those military health workers with daily average news checking of more than 10 times had significantly higher score than those who checked 3 to 10 times $(p=0.017)$ and $<3$ times $(p=0.014)$. There also existed a significant difference in the daily working hours $(p=0.019)$, daily sleep duration $(p=0.003)$, and sleep quality $(p<0.001)$ subgroups.

In terms of somatic symptoms, the score of female and doctor were significantly higher than male $(p=$ $0.008)$ and nurse $(p=0.011)$. Participants with junior $(p=0.004)$ and medium $(p=0.001)$ grade professional titles had significantly higher score than those with senior titles. There also existed a significant difference in the daily sleep duration $(p=0.001)$, and sleep quality $(p<0.001)$ subgroups. Results of hierarchical logistic regression analysis were presented in Table 4.Professional title $(p=0.010)$, daily sleep duration $(p=0.004)$, and sleep quality $(p<0.0001)$ were found to be significantly associated with PHQ-9 score. Age $(p=0.013)$ and sleep quality $(p=0.001)$ were associated with GAD-7 scores. Age $(p=0.017)$, daily sleep duration $(p=0.018)$, and sleep quality $(p<0.0001)$ were found to be significantly associated with PHQ-15 scores.

\section{Discussion}

The outbreak of COVID-19, which was regarded as a public health emergency, not only raised public health concerns but also caused tremendous psychological distress. At present, although the increase of confirmed and suspected cases of COVID-19 has been constrained in China, the first-line health workers still need to keep working. Military health workers who were among the first group to engage in the 
epidemic prevention experienced stern tests and more pressures due to the long work time, becoming the high risk group with mental health problems.

The prevalence of depression, generalized anxiety and somatic symptoms in military health workers was high, $37.6 \%, 32.5 \%$ and $50 \%$, respectively. Based on Liu and colleagues' study, it was approximately higher than the prevalence of depression (18.1\%) and anxiety (34.0\%) for the public[8]. There are three reasons to explain this result. Firstly, patients in the COVID19-specialized hospital were all COVID19confirmed cases that had older ages and more underlying diseases, such as cognitive disorders and psychotic symptoms. Secondly, military health workers were entrusted with this mission under critical circumstances. They needed to rescue patients at relatively unfamiliar places, with insufficient psychological and materials preparation. Lastly, the living condition and dwelling environment was poor. For instance, they had to travel a long distance from their residence to the hospitals, and they often had irregular meals and insufficient sleep due to heavy workload. However, it was worth noting that their prevalence of mental issues was lowered than that of health workers in the local hospitals during the COVID-19 outbreak. Huang and colleagues' study revealed that the prevalence of depression and anxiety of health workers in the local hospitals was as high as $50.7 \%$ and $44.7 \%$, respectively [9]. Meanwhile, our study shows that the $22.7 \%$ of military health workers with depression symptoms had comorbid anxiety and somatization, which was lower than that of primary care clinic patients in the United States [5]. Great tenacity and indomitableness of military medical workers may be picked out as possible causes, protecting their mental health to some extent.

Military health workers with lower grade professional title showed more depression symptoms, which are consistent with previous study [10], may be related to the following reasons. People with junior-grade professional title faced more challenges, such as economic pressure and limited societal resources. Also, in term of promotion, they need to consider other issues while treatingCOVID-19 patients, such as scientific researches, publishing papers and teaching tasks. Our study also shows that with the increase of age, more symptoms of anxiety and somatization appear in military health workers. These results indicated that older military health workers may endure more occupational exhaustion and family responsibilities.

The present study found depression, generalized anxiety and somatic symptoms were more prevalent in the military health workers with less sleep duration and poor sleep quality. This was in line with previous study done by Kobayashi and colleagues, showing poor sleep was highly associated with symptoms of depression, anxiety and PTSD[11]. Almost $81 \%$ military health workers had self-reported poor or ordinary sleep quality in this study. Living far from the hospital, time-consuming medical protection, generalized anxiety caused by severe epidemic situation, working in shifts leading to circadian rhythm disorder, and job burnout may have brought negative influences on the sleep quality.

Many powerful measures had been taken to maintain the mental health of the public in China. The national health commission of the PRC has issued guidelines for emergency psychological crisis intervention in COVID-19 [12]. In the guidelines, intervention subjects have been divided into four grades 
according to the degree of epidemic influence, of which military health workers were regarded as the firstgrade. In order to improve the mental health of military health workers in public health emergencies, some practical suggestions have been put forward based on the assessment of mental health conditions of military health workers and the analysis of related risk factors. Firstly, more attention should be paid to the specific groups, such as nurses and those with junior and medium grade professional titles, providing the support of "physiological-psychological-social" intervention. Secondly, it is not advisable to check information about COVID-19 frequently. Lastly, improvement of sleep quality and sleeping environment is needed. Mindfulness meditation intervention, relaxation training through virtual reality (VR), cognitive behavioral therapy for insomnia (CBTI) and sedative and hypnotic drugs may contribute to solving sleeping problems.

There are several limitations in the current study. Firstly, due to the sudden appearance of COVID-19, only a cross-sectional design was conducted. Secondly, the baseline mental health of military health workers before the outbreak of COVID-19 was lacking. Next, the relationship between mental health and other risk factors such as COVID-19 knowledge reserve and proportion of clinical classification of COVID-19 were not investigated. Lastly, results also needed to be explored in a larger sample size.

\section{Conclusion}

In conclusion, this study explored the prevalence of depression, generalized anxiety and somatic symptoms in military health workers during the COVID-19 outbreak. Moreover, related risk factors were investigated. Junior-grade professional title, older age, less sleep duration, and poor sleep quality have significant effects on the mental health of military health workers. Some suggestions aiming at military health workers have been provided in order to ensure their mental health, thus promoting military combat effectiveness.

\section{Abbreviations}

COVID-19: Coronavirus disease 2019; PHQ-9: Patient health questionnaire-9; GAD-7: Generalized anxiety disorder-7; PHQ-15: Patient health questionnaire-15; LSD: Least significance difference; PTSD: Post traumatic stress disorder; VR: Virtual reality; CBTI: Cognitive behavioral therapy for insomnia

\section{Declarations}

\section{Acknowledgements}

We acknowledge all participants of this project and investigators for collecting data.

\section{Funding}

This work was supported by Changzheng Hospital Pyramid Talent Project $₫ 2020 \rrbracket$ of Second Military Medical University and Research Project of Hubei Maternal and Child Health Hospital Guanggu District 
(2020-FYGG-017).

\section{Availability of data and materials}

All the relevant data and materials are presented in this article.

\section{Authors' contributions}

PX, BY, HY, ZF and ZQ participated in research design and drafted the manuscript. PX, ZF, RD, LF, HY and YX did literature search for citation. RD, ZF and YL designed the questionnaires. ZQ, YL, LF and YX participated in data analysis and data interpretation for the research. All the authors participated in discussion of the outcomes and offered their unique contribution to this research.

\section{Ethics approval}

For this survey, there is a positive vote by the ethics panel of the Medical Association Changzheng Hospital (No.2020SL010).All subjects in this study has given their informed consent.

\section{Consent for publication}

Not applicable.

\section{Competing interests}

There is no conflict of interests in this research.

\section{Author details}

${ }^{1}$ Department of Medical Psychology, Changzheng Hospital, Second Military Medical University, Shanghai 200005, China

${ }^{2}$ Hongkou District of Changhai Hospital, Second Military Medical University, Shanghai 200081, China

${ }^{3}$ Department of medical psychology, PLA Navy N0.905 Hospital, Second Military Medical University, Shanghai 200030, China

${ }^{4}$ Changzheng Hospital, Second Military Medical University, Shanghai 200005, China

${ }^{5}$ Department of Hyperbaric, Changhai Hospital, Second Military Medical University, Shanghai 200433, China

${ }^{6}$ Department of Radiotherapy, Changhai Hospital, Second Military Medical University, Shanghai 200433, China 
${ }^{7}$ Department of Critical Medicine, Changhai Hospital, Second Military Medical University, Shanghai 200433, China

\section{References}

1. Occupational injury insurance supports occupational injury. "protection umbrella" for prevention and rescue personnel in the fight against new coronavirus pneumonia.[2020-02-26].[http://www.nhc.gov. cn/xcs/fkdt/202001/0ffbb879ac1b4466b01de 8b40a1372fc.shtml].

2. Wang D, Hu B, Hu C, et al. Clinical Characteristics of 138 Hospitalized Patients With 2019 Novel Coronavirus-Infected Pneumonia in Wuhan, China. JAMA 2020.

3. Kang L, Li Y, Hu S, et al. The mental health of medical workers in Wuhan, China dealing with the 2019 novel coronavirus. The Lancet Psychiatry 2020; 7.

4. Panagioti M, Geraghty K, Johnson J, et al. Association Between Physician Burnout and Patient Safety, Professionalism, and Patient Satisfaction: A Systematic Review and Meta-analysis. JAMA Internal Medicine 2018; 178.

5. Löwe B, Spitzer R, Williams J, et al. Depression, anxiety and somatization in primary care: Syndrome overlap and functional impairment. Gen Hosp Psychiatry. 2008;30:191-9.

6. Koh D, Lim M, Chia S, et al. Risk Perception and Impact of Severe Acute Respiratory Syndrome (SARS) on Work and Personal Lives of Healthcare Workers in Singapore. Medical care. 2005;43:67682.

7. Lu YC, Shu BC, Chang YY, Lung FW. The mental health of hospital workers dealing with severe acute respiratory syndrome. Psychother Psychosom. 2006;75(6):370-5. doi:10.1159/000095443.

8. Leonhart R, Tang L, Pang Y, Li J, Song L, Fischer I, et al. (2016). Physical and psychological correlates of high somatic symptom severity in chinese breast cancer patients. Psycho-Oncology.

9. Teymoori A, Real R, Gorbunova A, et al. Measurement invariance of assessments of depression (PHQ-9) and anxiety (GAD-7) across sex, strata and linguistic backgrounds in a European-wide sample of patients after Traumatic Brain Injury. J Affect Disord. 2020;262:278-85.

10. Huang Y, Zhao N. Generalized anxiety disorder, depressive symptoms and sleep quality during COVID-19 epidemic in China: a web-based cross-sectional survey. MedRxiv 2020.

11. Kobayashi I, Boarts J, Delahanty D. Polysomnographically measured sleep abnormalities in PTSD. Psychophysiology. 2007;44:660-9.

12. Circular on Issuing Guiding Principles for Emergency Psychological Crisis Intervention for new coronavirus pneumonia. [2020-02-26]. [http://www.nhc.gov.cn/jkj/s3577/202001/6adc08b966594253b2b791be5c3b9467.shtml].

\section{Tables}

Due to technical limitations, tables are only available as a download in the supplemental files section. 


\section{Supplementary Files}

This is a list of supplementary files associated with this preprint. Click to download.

- Table4.xls

- Table1.xls

- Table3.xis

- Table2.xls 